\title{
Journal Club: MRI reveals acute inflammation in cortical lesions during early MS
}

Emily M. Bateman, BS, Wolfgang E. Schleicher, BS, Elana J. Smith, BS, David R. Sweet, BS, and Andrew D. Gaudet, PhD

Neurology ${ }^{\circledR}$ 2018;90:e724-e726. doi:10.1212/WNL.0000000000005001

\author{
Correspondence \\ Dr. Gaudet \\ andrew.gaudet@colorado. \\ edu
}

\begin{abstract}
Early multiple sclerosis is characterized by immune-associated demyelination of CNS axons. In a recent Neurology ${ }^{\circledR}$ article, Maranzano et al. evaluated MRI scans of patients with early multiple sclerosis to study the evolution of leukocortical lesions. Their novel data suggest that acute inflammation after blood-brain barrier leakage may contribute to gray matter cortical lesions in early multiple sclerosis.

Multiple sclerosis (MS) is a chronic neuroinflammatory and neurodegenerative disease that affects approximately 2.5 million people in the United States. ${ }^{1}$ Challenges with efficacy of existing drugs suggest the need for better understanding of mechanisms underlying MS. Demyelination of axons within the CNS, a hallmark of MS, is likely induced or worsened by inflammation. ${ }^{1}$ Due to technical limitations, white matter MS lesions have been easier to detect, but gray matter also appears susceptible to early inflammatory pathology. Indeed, leukocortical lesions, which are combined gray and white matter inflammatory lesions, and cortical demyelination can precede or co-present with meningeal inflammation. ${ }^{2}$ However, there remains an incomplete understanding about the temporal dynamics of leukocortical lesion development in MS.
\end{abstract}

In a recent article published in Neurology $y^{\circledR}$, Maranzano et al. ${ }^{3}$ compiled MRI scans of patients with early MS to better understand the evolution of these inflammatory cortical lesions (CLs). They reveal the dynamics of gray matter pathology during early MS, laying a foundation for identifying targets for ameliorating CL development and disease progression/severity. 


\section{Hypothesis and design}

Do patients with early MS present with inflammatory lesions involving blood-brain barrier (BBB) breakdown in the cortical gray matter? This study aimed to discover whether patients with early MS present with CLs enhanced with gadolinium, which would suggest leukocortical lesions (lesions associated with BBB damage). CLs are prevalent in many cases of endstage MS-but they could influence cortical pathology in early MS. In this observational, retrospective, longitudinal analysis, Maranzano et al. studied 1,188 MRI scans of 75 patients with early MS. Patients were scanned monthly over 12-24 months, enabling systematic study of individual CLs over time.

\section{Methods}

\section{Study design}

The study cohort was defined from individuals within the Betaseron vs Copaxone in Multiple Sclerosis with TripleDose Gadolinium and 3T MRI Endpoints (BECOME) study. ${ }^{3}$ Individuals with early relapsing-remitting MS and clinically isolated syndromes (CIS) suggestive of MS received one of 2 immunomodulatory treatments (interferon- $\beta-1 b$ or glatiramer acetate). For each MRI, individuals received a triple dose (i.e., $3 \times$ cumulative dose of a typical single dose, over 2 injections) of gadolinium to enhance lesion detection rate.

\section{MRI acquisition and analysis}

Individuals were scanned monthly for $1-2$ years ( $85 \%$ retention) to obtain T1-weighted images after gadolinium injection. Precontrast and postcontrast images revealed gadoliniumenhancing lesions. One specialist analyzed MRI scans for potential CLs by overlaying masks for enhancing lesions and cortex. Another specialist quantified binary CL voxel masks. Lesions were assessed in all 3 planes, and in linked precontrast and postcontrast injection images. Gadolinium-enhancing CLs were validated using stringent criteria, including minimum voxel size, hyperintensity in postcontrast T1-weighted image, and hyperintensity using another MRI sequence.

\section{Results}

Of the 1,188 scans analyzed during initial scan acquisition, 144 scans potentially contained gadolinium-enhancing lesions involving the cortex. Within these cases, 120 lesions affected the cortex (120 CLs out of 1,134 total lesions: 6\% of total enhancing lesions). Of these CLs, $95 \%$ were found to be type I leukocortical lesions (with clear involvement of subcortical white matter), whereas only 5\% were type II intracortical lesions (entirely within the gray matter). Remarkably, 27 of the 75 patients in the study demonstrated cortical enhancement, representing $36 \%$ of the patient population. Of particular interest to the investigators was the pattern of leukocortical lesions over time. Of the 52 lesions evaluated for longitudinal persistence, 27 (52\%) enhanced for less than 30 days, 6 (11\%) lasted between 30 and 60 days, 1 (2\%) lasted between 60 and 90 days, and 1 (2\%) lasted between 120 and 180 days. In addition to identifying the relatively transient nature of these lesions, radiologic evidence of lesion resolution was assessed. Using multiple MRI modalities of serial images, the authors found that $29 \%$ of enhancing lesions resolved completely without evidence of persistent damage on follow-up scans. Seventeen percent of lesions left subtle hyperintense signal that was only identifiable as an MS lesion when retrospectively compared to previous scans of larger lesions. Finally, 54\% of enhancing lesions that resolved remained notably hyperintense despite the absence of gadolinium.

\section{Interpretation}

Researchers detected enhancing lesions affecting the cortex and surrounding white matter. Gray matter lesions often present with $\mathrm{BBB}$ disruption. ${ }^{4}$ These findings suggest there may be acute inflammation in MS CL development. Future studies should explore how enhancing lesions relate to acute inflammation, other CL types, and neurodegeneration.

CLs have been recently implicated in end-stage MS using histology; however, it remained unclear whether CLs in early MS exhibit inflammation solely due to local CNS pathology or if these CLs also involve early BBB breakdown. This study reveals new information about CLs and whether acute inflammation occurs in CLs of patients with early MS.

\section{Strengths of the study}

\section{Study design}

The retrospective, longitudinal, observational analysis was a valuable choice for the study design. The longitudinal analysis of patients allowed for representation of CL progression in early MS, which is unique as most CL research is conducted postmortem. Monthly MRI scanning over 2 years with an $85 \%$ retention and $88 \%$ compliance rate supports the validity of the results.

\section{Triple gadolinium dosing strategy}

Three doses of gadolinium followed by a delay enabled effective MRI visualization of early MS CLs. This triple dosing improves sensitivity for visualizing immune cell infiltration in lesions with damaged $\mathrm{BBB}$ compared to standard-dose gadolinium MRI. ${ }^{5}$

\section{Stringent analysis criteria}

Evolution of the lesion enhancement was tracked through the presence or absence of gadolinium-enhancing regions in specific voxels with visible hyperintensity on T1- and T2-weighted imaging, fluid-attenuated inversion recovery, and proton density-weighted sequences. The use of combined masks and coregistered images effectively revealed CLs. Exclusion criteria were clearly stated.

\section{Novelty of results}

The results showed $36 \%$ of patients with early MS had gadolinium enhancement in the cortex at various time points throughout the study. 


\section{Study limitations}

\section{Image rating and evaluation}

Since only 2 (experienced) individuals analyzed 2 key aspects of the study, the rater's opinion could influence subsequent stages of the study. For example, distinguishing lesions that had "sufficiently large" cortical involvement is partially subjective and could alter false-negative/false-positive rates. Alternatively, 2-3 independent raters could identify gadolinium-enhancing lesions and CLs. This would add rigor and minimize bias.

\section{Disease spectrum and treatment arms}

Clearly, recruiting sufficient patients who are clinically similar is challenging. Because this study uses a timeline approach for analysis, however, the results could be confounded by including multiple disease states (i.e., CIS, relapsing-remitting MS). For example, the inflammatory (and radiologic) pattern between the transition between CIS and diagnosed MS may differ from that of relapsing-remitting MS: in one study, patients with CIS showed no evidence of brain atrophy year to year, whereas patients with relapsing-remitting MS (either direct relapsing-remitting MS or converted from CIS) lost $\sim 0.2 \%$ of brain parenchymal volume per year. ${ }^{6}$ Further, CLs are more prevalent in patients with relapsing-remitting MS (CLs found in 64\% of patients' brains) than in those with CIS (CLs in 36\% of patients' brains). ${ }^{7}$

In addition, combining patients on 2 separate immunomodulatory treatment regimens into one group for internal analysis is a potential confound. While the BECOME study demonstrated no quantitative differences in active lesions, it did not describe the spatial localization of lesions between the interferon- $\beta$ and glatiramer acetate groups. ${ }^{8}$ Therefore, it would be beneficial to understand whether either treatment arm had more leukocortical lesions. Additional analyses could indicate whether the treatments altered temporal dynamics of lesions (i.e., acute vs persistent lesions).

\section{Underestimation of inflammatory lesions}

Because all patients in the study were treated by immunomodulators, the inflammatory lesions presented may underestimate the underlying pathology. In early articles describing the use of interferon- $\beta$ and glatiramer acetate in treating MS, one critical readout of efficacy was the attenuation of lesions on MRI. ${ }^{9}$ If, as the authors show, there are transient, acute inflammatory lesions that resolve over time, this effect may be enhanced in patients who are not taking immunomodulatory drugs. Therefore, one could examine data from previously performed placebo-controlled trials to assess the existence and persistence of early CLs in MS.

\section{Use of conventional imaging techniques}

Normal-appearing brain parenchyma, as assessed by conventional imaging, can in fact have pathologies that are revealed using more advanced technologies. For instance, compared to 3T MRI (used in this study), ultra-high-field 7T MRI detects twice the number of CLs; further, other advanced techniques such as $3 \mathrm{D}$ double inversion recovery and phase difference- enhanced imaging could help improve discovery of CLs. ${ }^{10}$ Future studies on early MS could further enhance CL identification by using cutting-edge imaging techniques.

While the damaging effects of MS on white matter have been extensively characterized, the relative contributions of gray matter lesions to MS pathology have been radiologically elusive. Using longitudinal gadolinium-enhanced MRI from the BECOME study, Maranzano et al. provide evidence that early leukocortical lesions occur during early MS, many of which disappear with no evidence of pathology on follow-up scans. These novel results suggest that early MS gray matter pathology may be related to BBB breakdown. This work provides the foundation for future studies exploring how the distribution, timing, and quantity of CLs may inform novel mechanisms of disease or markers of prognosis in MS.

\section{Author contributions}

Emily M. Bateman: drafting/revising the manuscript, analysis or interpretation of data, accepts responsibility for conduct of research and final approval, study supervision. Wolfgang E. Schleicher: drafting/revising the manuscript, accepts responsibility for conduct of research and final approval. Elana J. Smith: analysis or interpretation of data, accepts responsibility for conduct of research and final approval, acquisition of data, statistical analysis. David R. Sweet: drafting/revising the manuscript, analysis or interpretation of data, accepts responsibility for conduct of research and final approval. Andrew D. Gaudet: drafting/revising the manuscript, study concept or design, accepts responsibility for conduct of research and final approval, study supervision, obtaining funding.

\section{Study funding}

A.D.G. is supported by a grant from the Wings for Life Foundation.

\section{Disclosure}

The authors report no disclosures relevant to the manuscript. Go to Neurology.org/N for full disclosures.

\section{References}

1. Dargahi N, Katsara M, Tselios T, et al. Multiple sclerosis: immunopathology and treatment update. Brain Sci 2017;7.

2. Popescu BF, Lucchinetti CF. Meningeal and cortical grey matter pathology in multiple sclerosis. BMC Neurol 2012;12:11.

3. Maranzano J, Rudko DA, Nakamura K, et al. MRI evidence of acute inflammation in leukocortical lesions of patients with early multiple sclerosis. Neurology 2017;89:714-721.

4. Calabrese M, Magliozzi R, Ciccarelli O, Geurts JJ, Reynolds R, Martin R. Exploring the origins of grey matter damage in multiple sclerosis. Nat Rev Neurosci 2015;16:147-158.

5. Filippi M, Yousry T, Campi A, et al. Comparison of triple dose versus standard dose gadolinium-DTPA for detection of MRI enhancing lesions in patients with MS. Neurology 1996;46:379-384.

6. Fisher E, Lee JC, Nakamura K, Rudick RA. Gray matter atrophy in multiple sclerosis: a longitudinal study. Ann Neurol 2008;64:255-265.

7. Calabrese M, De Stefano N, Atzori M, et al. Detection of cortical inflammatory lesions by double inversion recovery magnetic resonance imaging in patients with multiple sclerosis. Arch Neurol 2007;64:1416-1422.

8. Cadavid D, Wolansky LJ, Skurnick J, et al. Efficacy of treatment of MS with IFNbeta$1 \mathrm{~b}$ or glatiramer acetate by monthly brain MRI in the BECOME study. Neurology 2009;72:1976-1983.

9. The IFNB Multiple Sclerosis Study Group. Interferon beta-1b is effective in relapsing remitting multiple sclerosis: I: clinical results of a multicenter, randomized, doubleblind, placebo-controlled trial. Neurology 1993;43:655-661.

10. Mahajan KR, Ontaneda D. The role of advanced magnetic resonance imaging techniques in multiple sclerosis clinical trials. Neurotherapeutics 2017;14:905-923. 


\section{Neurology}

Journal Club: MRI reveals acute inflammation in cortical lesions during early MS Emily M. Bateman, Wolfgang E. Schleicher, Elana J. Smith, et al.

Neurology 2018;90;e724-e726

DOI 10.1212/WNL.0000000000005001

This information is current as of February 19, 2018

Updated Information \& Services

References

Citations

Subspecialty Collections

Permissions \& Licensing

Reprints including high resolution figures, can be found at: http://n.neurology.org/content/90/8/e724.full

This article cites 9 articles, 4 of which you can access for free at: http://n.neurology.org/content/90/8/e724.full\#ref-list-1

This article has been cited by 1 HighWire-hosted articles: http://n.neurology.org/content/90/8/e724.full\#\#otherarticles

This article, along with others on similar topics, appears in the following collection(s):

Autoimmune diseases

http://n.neurology.org/cgi/collection/autoimmune_diseases

Multiple sclerosis

http://n.neurology.org/cgi/collection/multiple_sclerosis

Information about reproducing this article in parts (figures,tables) or in its entirety can be found online at:

http://www.neurology.org/about/about_the_journal\#permissions

Information about ordering reprints can be found online:

http://n.neurology.org/subscribers/advertise

Neurology ${ }^{\circledR}$ is the official journal of the American Academy of Neurology. Published continuously since 1951, it is now a weekly with 48 issues per year. Copyright @ 2018 American Academy of Neurology. All rights reserved. Print ISSN: 0028-3878. Online ISSN: 1526-632X.

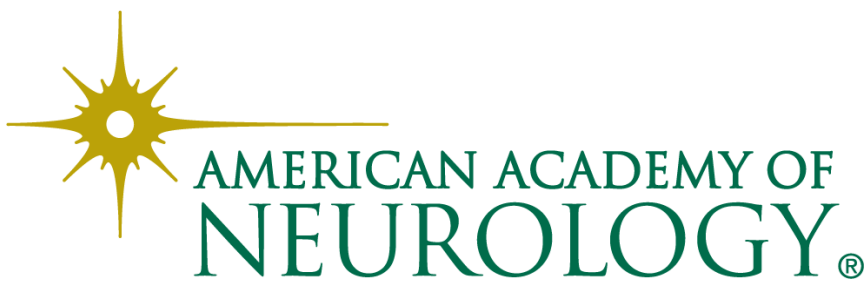

